\title{
Using topology of molecular solvation to enhance biomolecular structural refinement
}

\author{
George M. Giambasu, David A. Case \\ Rutgers University
}

Binding of ions and water to specific sites is intimately linked with how supramolecular assemblies form and how biomolecules fold and behave in a test-tube, crystal or cell. The new models based on 3D-RISM and new topological analysis methods that I developed are powerful computational tools for prediction of site-specific binding to a wide array of biological systems and molecules relevant to supramolecular chemistry. Herein, these new tools are applied to crystallographic refinement of a variety of nucleic acids molecules and ion channels using the the AMBER molecular simulation suite coupled with the PHENIX general purpose crystallographic structure refinement program. 